\title{
Isoperimetric inequalities for cartesian products of graphs
}

\author{
F. R. K. Chung \\ University of Pennsylvania \\ Philadelphia 19104
}

\author{
Prasad Tetali \\ School of Mathematics \\ Georgia Inst. of Technology \\ Atlanta GA 30332-0160
}

\begin{abstract}
We give a characterization for isoperimetric invariants, including the Cheeger constant and the isoperimetric number of a graph. This leads to an isoperimetric inequality for the cartesian products of graphs.
\end{abstract}

\section{Introduction}

For a graph $G$ and a subset $S$ of vertex set $V(G)$ of $G$, the edge-boundary $\partial S$ of $S$ consists of all edges with exactly one endpoint in $S$ :

$$
\partial S=\{\{u, v\} \in E(G): u \in S \text { and } v \notin S\}
$$

Let $\bar{S}$ denote the complement of $S$, i.e., $\bar{S}=V-S$. Clearly, $\partial S=\partial \bar{S}=E(S, \bar{S})$ where $E(A, B)$ denotes the set of edges with one endpoint in $A$ and one endpoint in $B$.

There are two types of isoperimetric invariants which are often mentioned in the literature:

(1) The Cheeger constant of $G$ is defined (see $[5,6]$ ) to be

$$
h(G)=\inf _{S} \frac{|E(S, \bar{S})|}{\min (\operatorname{vol} S, \operatorname{vol} \bar{S})} .
$$

where the volume of $S$, denoted by vol $S$, is the sum of all degrees $d_{x}$ for $x \in S$.

(2) The isoperimetric number of $G$ is defined (see [11]) to be

$$
i(G)=\inf _{S} \frac{|E(S, \bar{S})|}{\min (|S|,|\bar{S}|)} .
$$


For a weighted graph $G$ with vertex-weight $w(v)$, for $v \in V(G)$ and edge-weight $w(u, v)=w(v, u)$, we can define the isoperimetric invariant $h(G, w)$ :

$$
h(G, w)=\inf _{S} \frac{\sum_{\{u, v\} \in E(S, \bar{S})} w(u, v)}{\min \left(\sum_{u \in S} w(u), \sum_{v \in \bar{S}} w(v)\right)} .
$$

We say the weight function $w$ is consistent if

$$
\sum_{u} w(u, v)=w(v)
$$

For example, the Cheeger constant is obtained by using the weight function $w_{0}(u, v)=1$ for any edge $\{u, v\}$ and $w_{0}(v)=d_{v}$ for any vertex $v$. Clearly, $w_{0}$ is consistent. On the other hand, the isoperimetric number is just $i(G)=h\left(G, w_{1}\right)$ where the weight function $w_{1}$ satisfies $w_{1}(u, v)=1$ for any edge $\{u, v\}$ and $w_{1}(v)=1$ for any vertex $v$. Of course, $w_{1}$ is not consistent. We note that graphs with consistent weight functions correspond in a natural way to random walks and reversible Markov chains. Namely, for a graph with a consistent weight function $w$, we can define the random walk with transition probability of moving from a vertex $u$ to each of its neighbors $v$ to be

$$
P(u, v)=\frac{w(u, v)}{w(u)}
$$

For further discussions, the reader is referred to $[1,6]$.

First, we will establish the following characterizations for $h(G, w)$.

Theorem 1 For a graph $G$ with weight function $w$, the isoperimetric invariant $h(G, w)$ of a graph G satisfies

$$
h(G, w)=\inf _{f \neq 0} \sup _{c \in R} \frac{\sum_{x \sim y}|f(x)-f(y)| w(x, y)}{\sum_{x \in V}|f(x)-c| w(x)}
$$

where $f$ ranges over all $f: V \rightarrow R$ which are not identically zero.

As an immediate consequence of Theorem 1, we have the following characterization for the Cheeger constant and the isoperimetric number.

$$
h(G)=\inf _{f \neq 0} \sup _{c \in R} \frac{\sum_{x \sim y}|f(x)-f(y)|}{\sum_{x \in V}|f(x)-c| d_{x}}
$$


where $f$ ranges over all $f: V \rightarrow R$ which are not identically zero. The isoperimetric number $i(G)$ of a graph $G$ satisfies

$$
i(G)=\inf _{f \neq 0} \sup _{c \in R} \frac{\sum_{x \sim y}|f(x)-f(y)|}{\sum_{x \in V}|f(x)-c|}
$$

where $f$ ranges over all $f: V \rightarrow R$ which are not identically zero.

For two graphs $G$ and $H$, the cartesian product $G H$ has vertex set $V(G) \times V(H)$ with $(u, v)$ adjacent to $\left(u^{\prime}, v^{\prime}\right)$ if and only if $u=u^{\prime}$ and $v$ is adjacent to $v^{\prime}$ in $H$, or $v=v^{\prime}$ and $u$ is adjacent to $u^{\prime}$ in $G$. There are many research papers examining the isoperimetric number for various graphs. For example, Wang and Wang [12] and Bollobas and Leader [3, 4] studied the isoperimetric number for grids (which are cartesian products of paths) and torus (which are cartesian products of cycles). In particular, there is a large literature examining the isoperimetric problems for $n$-cube which is just the $n$-fold cartesian product of a single edge (see Harper [7], Lindsey [10], Bernstein [2] and Hart [8]). Also, Mohar [11] considered the special case of the cartesian product of a graph and a complete graph. In this paper, we consider cartesian products of general graphs. We will establish the relationship between the isoperimetric invariants of graphs and their cartesian products. The proof is by using a variation of the above characterization in Theorem 1 .

Theorem 2 The isoperimetric number of the cartesian product of $G_{1}, G_{2}, \ldots, G_{k}$ satisfies

$$
\begin{aligned}
\min \left(i\left(G_{1}\right), i\left(G_{2}\right), \cdots, i\left(G_{k}\right)\right) & \geq i\left(G_{1} G_{2} \cdots G_{k}\right) \\
& \geq \frac{1}{2} \min \left(i\left(G_{1}\right), i\left(G_{2}\right), \cdots, i\left(G_{k}\right)\right)
\end{aligned}
$$

We remark that a Markov chains analog of Theorem 2 was proved in [9] with a somewhat complicated proof and a weaker constant (2/9 instead of $1 / 2)$.

We will consider a cartesian product of weighted graphs with consistent weight functions. For two weighted graphs $G$ and $G^{\prime}$, with weight functions $w, w^{\prime}$, respectively, the weighted cartesian product $G \otimes G^{\prime}$ has vertex set $V(G) \times V\left(G^{\prime}\right)$ with weight function $w \otimes w^{\prime}$ defined as follows: For an edge $\{u, v\}$ in $E(G)$, we define $w \otimes w^{\prime}\left(\left(u, v^{\prime}\right),\left(v, v^{\prime}\right)\right)=w(u, v) w^{\prime}\left(v^{\prime}\right)$ and for an edge $\left\{u^{\prime}, v^{\prime}\right\}$ in $E\left(G^{\prime}\right)$, we define $w \otimes w^{\prime}\left(\left(u, u^{\prime}\right),\left(u, v^{\prime}\right)\right)=w(u) w^{\prime}\left(u^{\prime}, v^{\prime}\right)$. We require $w \otimes w^{\prime}$ to be consistent. Clearly, for a vertex $x=(u, v)$ in $G \otimes G^{\prime}$, the weight of $x$ in $G \otimes G^{\prime}$ is exactly $2 w(u) w^{\prime}(v)$.

In general, for graphs $G_{i}$ with consistent weight functions $w_{i}, i=1, \cdots, k$, the weighted cartesian product $G_{1} \otimes \cdots \otimes G_{k}$ has vertex set $V(G) \otimes \cdots \otimes V\left(G_{k}\right)$ with a consistent weight function $w_{1} \otimes \cdots \otimes w_{k}$ 
defined as follows: For an edge $\{u, v\}$ in $E\left(G_{i}\right)$, the edges joining $\left(v_{1}, \cdots, v_{i-1}, u, v_{i+1}, \cdots, v_{k}\right)$ and $\left(v_{1}, \cdots, v_{i-1}, v, v_{i+1}, \cdots, v_{k}\right)$ has weight $w_{1}\left(v_{1}\right) \cdots w_{i-1}\left(v_{i-1}\right) w_{i}(u, v) w_{i+1}\left(v_{i+1}\right), \cdots, w_{k}\left(v_{k}\right)$.

For a graph $G$, the natural consistent weight function associated with $G$ is with edge weight 1 and vertex weight $d_{x}$ for any vertex $x$. (Here, we consider a graph as a weighted graph with the natural consistent weight function.)

Theorem 3 The Cheeger constant of a weighted cartesian product of $G_{1}, G_{2}, \ldots, G_{k}$ satisfies

$$
\begin{aligned}
\min _{j}\left(h\left(G_{j}\right)\right) & \geq h\left(G_{1} \otimes G_{2} \otimes \cdots \otimes G_{k}\right) \\
& \geq \frac{1}{2 k} \min _{j}\left(h\left(G_{j}\right)\right)
\end{aligned}
$$

We remark that the weighted cartesian product of graphs corresponds to the cartesian product of random walks on graphs. Suppose $G_{1}, \cdots, G_{k}$ are weighted graphs with the vertex set $V\left(G_{i}\right)$. Each $G_{i}$ associates with a random walk with transition probability $P_{i}$ as defined as in 4 . The cartesian product of the random walks can be defined as follows: At the vertex $\left(v_{1}, \cdots, v_{k}\right)$, first choose a random "direction" $i$, between 1 and $k$, each with probability $1 / k$. Then move to the vertex $\left(v_{1}, \cdots, v_{i-1}, u_{i}, v_{i+1}, \cdots, v_{k}\right)$ according to $P_{i}$. In other words,

$$
P\left(\left(v_{1}, \cdots, v_{i-1}, v_{i}, v_{i+1}, \cdots, u_{k}\right),\left(v_{1}, \cdots, v_{i-1}, u_{i}, v_{i+1}, \cdots, v_{k}\right)\right)=\frac{1}{k} P\left(v_{i}, u_{i}\right)
$$

\section{A characterization of the isoperimetric invariant}

In this section, we will give the proof of Theorem 1 for a graph $G$ with a weight function $w$ by showing:

$$
h(G, w)=\inf _{f \neq 0} \sup _{c \in R} \frac{\sum_{x \sim y}|f(x)-f(y)| w(x, y)}{\sum_{x \in V}|f(x)-c| w(x)}
$$

Proof of Theorem 1: We choose $c$ such that

$$
\sum_{x: f(x)<c} w(x) \leq \sum_{x: f(x) \geq c} w(x)
$$

and

$$
\sum_{x: f(x) \leq c} w(x) \leq \sum_{x: f(x)>c} w(x)
$$


Let $g=f-c$. We consider

$$
\tilde{g}(\sigma)=\sum_{g(x) \leq \sigma<g(y)} w(x, y)
$$

Then we have

$$
\begin{aligned}
\sum_{x \sim y}|f(x)-f(y)| w(x, y)= & \int_{-\infty}^{\infty} \tilde{g}(\sigma) d \sigma \\
= & \int_{-\infty}^{0} d \sigma \frac{\tilde{g}(\sigma)}{\sum_{g(x)<\sigma} w(x)} \sum_{g(x)<\sigma} w(x) \\
& +\int_{0}^{\infty} d \sigma \frac{\tilde{g}(\sigma)}{\sum_{g(x)>\sigma} w(x)} \sum_{g(x)>\sigma} w(x) \\
\geq & h(G, w)\left(\int_{-\infty}^{0} d \sigma \sum_{g(x)<\sigma} w(x)+\int_{0}^{\infty} d \sigma \sum_{g(x)>\sigma} w(x)\right) \\
= & h(G, w) \sum_{x \in V}|f(x)-c| w(x)
\end{aligned}
$$

In the opposite direction, suppose $X$ is a subset of $V$ satisfying

$$
h(G, w)=\frac{\sum_{\{x, y\} \in E(X, \bar{X})} w(x, y)}{\sum_{x \in X} w(x)}
$$

We consider the following characteristic function $\chi$ of $X$ :

$$
\chi(x)= \begin{cases}1 & \text { if } x \in X \\ -1 & \text { otherwise }\end{cases}
$$

Then we have, for any $C$,

$$
\sup _{C} \frac{\sum x \sim y|\chi(x)-\chi(y)| w(x, y)}{\sum_{x \in V}|\chi(x)-C| w(x)}=\sup _{C} \frac{\sum_{\{x, y\} \in E(X, \bar{X})} 2 w(x, y)}{(1-C) \sum_{x \in X} w(x)+(1+C) \sum_{x \in \bar{X}} w(x)}
$$

The supremum is achieved when $C=-1$, and we have

$$
\sup _{C} \frac{\sum x \sim y|\chi(x)-\chi(y)| w(x, y)}{\sum_{x \in V}|\chi(x)-C| w(x)}=h(G, w)
$$


Therefore, we have

$$
h(G, w) \geq \inf _{f \neq 0} \sup _{c \in R} \frac{\sum_{x \sim y}|f(x)-f(y)| w(x, y)}{\sum_{x \in V}|f(x)-c| w(x)}
$$

and Theorem 1 is proved.

To derive the isoperimetric relationship between graphs and their cartesian products, we need the following variation of Theorem 1 :

Corollary 1 For a graph $G$, we have

$$
h(G, w) \geq \inf _{f} \frac{\sum_{x \sim y}|f(x)-f(y)| w(x, y)}{\sum_{x \in V}|f(x)| w(x)} \geq \frac{1}{2} h(G, w)
$$

where $f: V(G) \rightarrow R$ satisfies

$$
\sum_{x \in V} f(x) w(x)=0
$$

Proof: ¿From Theorem 1, we already have

$$
h(G, w) \geq \inf _{f} \frac{\sum_{x \sim y}|f(x)-f(y)| w(x, y)}{\sum_{x \in V}|f(x)| w(x)}
$$

for $f$ satisfies (8). It remains to prove the second part of the inequality. Suppose we define $c$ as in the proof of Theorem 1. If $c \leq 0$, then we have

$$
\begin{aligned}
\sum_{x}|f(x)-c| w(x) & \geq \sum_{x: f(x) \geq 0}|f(x)-c| w(x) \\
& \geq \sum_{x: f(x) \geq 0}|f(x)| w(x) \\
& \geq \frac{1}{2} \sum_{x}|f(x)| w(x) \quad \text { by }(7) .
\end{aligned}
$$

Similarly, if $c \geq 0$, then we have

$$
\begin{aligned}
\sum_{x}|f(x)-c| w(x) & \geq \sum_{x: f(x) \leq 0}|f(x)-c| w(x) \\
& \geq \sum_{x: f(x) \leq 0}|f(x)| w(x) \\
& \geq \frac{1}{2} \sum_{x}|f(x)| w(x) \quad \text { by }(7) .
\end{aligned}
$$

Therefore we have

$$
\inf _{f} \frac{\sum_{x \sim y}|f(x)-f(y)| w(x, y)}{\sum_{x \in V}|f(x)| w(x)} \geq \frac{1}{2} h(G, w)
$$

This completes the proof of the corollary. 


\section{An isoperimetric inequality for Cartesian products}

We first derive Theorem 2 concerning the isoperimetric number of the cartesian product of graphs $G_{j}, j=1, \ldots, k$. (This is the case with the weight function $w(v)=1$ for each vertex $v$.)

$$
\begin{aligned}
\min \left(i\left(G_{1}\right), i\left(G_{2}\right), \cdots, i\left(G_{k}\right)\right) & \geq i\left(G_{1} G_{2} \cdots G_{k}\right) \\
& \geq \frac{1}{2} \min \left(i\left(G_{1}\right), i\left(G_{2}\right), \cdots, i\left(G_{k}\right)\right)
\end{aligned}
$$

Proof of Theorem 2: Without loss of generality, we assume that

$$
i\left(G_{1}\right) \leq i\left(G_{j}\right), \text { for } j=1, \ldots, k \text {. }
$$

It is easy to see that

$$
i\left(G_{1} G_{2} \cdots G_{k}\right) \leq i\left(G_{1}\right)
$$

since we can choose a function $h: V(G) \times V(H) \rightarrow R$ by setting

$$
h\left(v_{1}, \ldots, v_{k}\right)=f\left(v_{1}\right)
$$

where $f: V(G) \rightarrow R$ is a function achieving $i\left(G_{1}\right)$ in $(7)$.

To prove

$$
i\left(G_{1} G_{2} \cdots G_{k}\right) \geq \frac{1}{2} \min \left(i\left(G_{1}\right), i\left(G_{2}\right), \cdots, i\left(G_{k}\right)\right)
$$

we consider a function $g: V\left(G_{1}\right) \times \cdots \times V\left(G_{k}\right) \rightarrow R$ which achives the value $i\left(G_{1} G_{2} \cdots G_{k}\right)$ in $(7)$. For vertices $v_{1} \in V\left(G_{1}\right), \ldots, v_{k} \in V\left(G_{k}\right)$, we consider functions $\bar{g}_{j}$ for $j=1, \ldots, k$.

$$
\bar{g}_{j}\left(v_{1}, \cdots, v_{k}\right)=\frac{\sum_{u_{1}, \cdots, u_{j}} g\left(u_{1}, \cdots, u_{j}, v_{j+1}, \cdots, v_{k}\right)}{\left|V\left(G_{1}\right)\right| \cdots\left|V\left(G_{j}\right)\right|}
$$

Note that

$$
\bar{g}_{j}\left(v_{1}, \cdots, v_{k}\right)=\frac{1}{\left|V\left(G_{j}\right)\right|} \sum_{u_{j}} \bar{g}_{j-1}\left(v_{1}, \cdots, v_{j-1}, u_{j}, v_{j+1}, \cdots, v_{k}\right)
$$

In particular, $\bar{g}_{k}$ is a constant function, namely, the average of all values of $g$. We have

$$
i\left(G_{1} G_{2} \cdots G_{k}\right) \geq \frac{I_{1}+\cdots+I_{k}}{\sum_{v_{1}, \cdots, v_{k}}\left|g\left(v_{1}, \cdots, v_{k}\right)-\bar{g}_{k}\right|}
$$


where

$$
I_{1}=\sum_{v_{2}, \cdots, v_{k}} \sum_{v_{1} \sim v_{1}^{\prime}}\left|g\left(v_{1}, v_{2}, \cdots, v_{k}\right)-g\left(v_{1}^{\prime}, v_{2}, \cdots, v_{k}\right)\right|
$$

and

$$
\begin{aligned}
& I_{j}=\sum_{v_{1}, \cdots, v_{j-1}, v_{j+1}, \cdots, v_{k}} \sum_{v_{j} \sim v_{j}^{\prime}} \mid g\left(v_{1}, \cdots, v_{j-1}, v_{j}, v_{j+1}, \cdots, v_{k}\right) \\
& -g\left(v_{1}, \cdots, v_{j-1}, v_{j}^{\prime}, v_{j+1}, \cdots, v_{k}\right) \mid
\end{aligned}
$$

Using Corollary 1, we have

$$
I_{1} \geq \frac{1}{2} \sum_{v_{2}, \cdots, v_{k}} i\left(G_{1}\right) \sum_{v_{1}}\left|g\left(v_{1}, v_{2}, \cdots, v_{k}\right)-\bar{g}_{1}\left(v_{1}, v_{2}, \cdots, v_{k}\right)\right|
$$

By using the definition of $\bar{g}_{j-1}$ and the triangle inequality, we have, for $j \geq 2$,

$$
\begin{aligned}
& I_{j} \geq\left|V\left(G_{1}\right)\right| \cdots\left|V\left(G_{j-1}\right)\right| \sum_{v_{j+1}, \cdots, v_{k}} \sum_{v_{j} \sim v_{j}^{\prime}} \quad \mid \bar{g}_{j-1}\left(v_{1}, \cdots, v_{j-1}, v_{j}, v_{j+1}, \cdots, v_{k}\right) \\
&-\bar{g}_{j-1}\left(v_{1}, \cdots, v_{j-1}, v_{j}^{\prime}, v_{j+1}, \cdots, v_{k}\right) \mid
\end{aligned}
$$

Now, applying the second part of the inequality in Corollary 1 for each copy of $G_{j}$, and using (8), we get

$$
\begin{aligned}
I_{j} & \geq \frac{1}{2} i\left(G_{j}\right)\left|V\left(G_{1}\right)\right| \cdots\left|V\left(G_{j-1}\right)\right| \sum_{v_{j+1}, \cdots, v_{k}} \sum_{v_{j}}\left|\bar{g}_{j-1}\left(v_{1}, \cdots, v_{k}\right)-\bar{g}_{j}\left(v_{1}, \cdots, v_{k}\right)\right| \\
& =\frac{1}{2} i\left(G_{j}\right) \sum_{v_{1}, \cdots, v_{k}}\left|\bar{g}_{j-1}\left(v_{1}, \cdots, v_{k}\right)-\bar{g}_{j}\left(v_{1}, \cdots, v_{k}\right)\right|
\end{aligned}
$$

where we used the fact that $\bar{g}_{j-1}$ and $\bar{g}_{j}$ do not depend on the particular choice of the first $j-1$ variables.

We also note that

$$
\begin{aligned}
& \sum_{v_{1}, \cdots v_{k}}\left|g\left(v_{1}, \cdots, v_{k}\right)-g_{k}\right| \leq \sum_{v_{1}, \cdots v_{k}}\left|g\left(v_{1}, \cdots, v_{k}\right)-g_{1}\left(v_{1}, \cdots, v_{k}\right)\right|+\cdots \\
& +\left|g_{k-1}\left(v_{1}, \cdots, v_{k}\right)-g_{k}\right|
\end{aligned}
$$

So, by comparing term by term, Theorem 2 is proved. 
Sketch of Theorem 3:

The analogus version for weighted cartesian product can be proved in essentially the same way (with the extra complexity of notation). Here, we will describe part of the proof which illustrates the qualitative difference from the proof of Theorem 2 and omit the rest of the proof of Theorem 3.

Without loss of generality, we assume $h\left(G_{1}\right)=h\left(G_{j}\right)$ for all $j$. Then it follows from the proof above that

$$
\begin{aligned}
I_{j}=\sum_{v_{1}, \ldots, v_{j-1}, v_{j+1}, \ldots, v_{k}} \sum_{v_{j} \sim v_{j}^{\prime}} \mid g\left(v_{1}, \ldots, v_{j-1}, v_{j}, v_{j+1}, \ldots, v_{k}\right) \\
\quad-g\left(v_{1}, \ldots, v_{j-1}, v_{j}^{\prime}, v_{j+1}, \ldots, v_{k}\right) \mid d_{v_{1}} \ldots d_{v_{i-1}} w\left(v_{j}, v_{j}^{\prime}\right) d_{v_{j+1}} \ldots d_{v_{k}}
\end{aligned}
$$

Therefore, we have

$$
\begin{aligned}
h\left(G_{1} \otimes \cdots \otimes G_{k}\right) & \geq \frac{I_{1}+I_{2}+\cdots+I_{k}}{\sum_{v_{1}, \cdots, v_{k}}\left|g\left(v_{1}, \cdots, v_{k}\right)-\bar{g}_{k}\right| d_{\left(v_{1}, \ldots, v_{k}\right)}} \\
& \geq \frac{\frac{1}{2} h\left(G_{1}\right) \sum_{j=1}^{k} \sum_{v_{1}, \cdots, v_{k}}\left|\bar{g}_{j-1}\left(v_{1}, \cdots, v_{k}\right)-\bar{g}_{j}\left(v_{1}, \cdots, v_{k}\right)\right| d_{v_{1}} \ldots d_{v_{j}}}{\sum_{v_{1}, \cdots, v_{k}}\left|g\left(v_{1}, \cdots, v_{k}\right)-\bar{g}_{k}\right| d_{\left(v_{1}, \ldots, v_{k}\right)}}
\end{aligned}
$$

where $\bar{g}_{0}=g$, and $d_{\left(v_{1}, \ldots, v_{k}\right)}$ represents the degree of the vertex $\left(v_{1}, \ldots, v_{k}\right)$ in the product graph. Thus

$$
\begin{aligned}
h\left(G_{1} \otimes \cdots \otimes G_{k}\right) & \geq \frac{1}{2} h\left(G_{1}\right) \frac{\sum_{j=1}^{k} \sum_{v_{1}, \cdots, v_{k}}\left|\bar{g}_{j-1}\left(v_{1}, \cdots, v_{k}\right)-\bar{g}_{j}\left(v_{1}, \cdots, v_{k}\right)\right| d_{v_{1}} \ldots d_{v_{k}}}{\sum_{v_{1}, \cdots, v_{k}}\left|g\left(v_{1}, \cdots, v_{k}\right)-\bar{g}_{k}\right| d_{v_{1}} \ldots d_{v_{k}}} \\
& \geq \frac{1}{2 k} h\left(G_{1}\right),
\end{aligned}
$$

by, again, the triangle inequality.

\section{References}

[1] D. Aldous, "Reversible Markov chains and random walks on graphs," book in preparation.

[2] A. J. Berstein, Maximally connected arrays on the n-cube, SIAM J. Appl. Math. 15 (1967) 1485-1489. 
[3] Bela Bollobás and I. Leader, Edge-isoperimetric inequalities in the grid, Combinatorica 11(1991) 299-314.

[4] Bela Bollobás and I. Leader, An isoperimetric inequality on the discrete torus, SIAM J. Disc. Math. 3 (1990) 32-37.

[5] J. Cheeger, A lower bound for the smallest eigenvalue of the Laplacian, Problems in Analysis, (R. C. Gunning, ed.) Princeton Univ. Press (1970) 195-199.

[6] F. R. K. Chung, Spectral Graph Theory, CMBS Lecture Notes, 1996, AMS Publication.

[7] L. H. Harper, Optimal numberings and isoperimetric problems on graphs, J. of Comb. Theory 1 (1966), 385-393.

[8] S. Hart, A note on the edges of the n-cube, Discrete Math. 14 (1976), 157-163.

[9] C. Houdré and P. Tetali, Isoperimetric constants for product Markov chains and graph products, preprint (January, 1995).

[10] J. H. Lindsey, Assignment of numbers to vertices, Amer. Math. Monthly, 71 (1964) 508-516.

[11] B. Mohar, Isoperimetric number of graphs, J. of Comb. Theory (B) 47 (1989), 274-291.

[12] D. -L. Wang and P. Wang, Discrete isoperimetric problems, SIAM J. Appl. Math. 32 (1977) 860-870. 